\title{
Recidiva local nos sarcomas de tecidos moles: fatores prognósticos
}

\section{Local recurrence in soft tissue sarcoma: prognostic factors}

luiz Eduardo Moreira Teixeira"; Ivana Duval Araújo; Marco Antônio Percope de Andrade33; Rogério Andrade Gomes; Paulo Guilherme de Oliveira Salles ${ }^{5}$; Daniel Ferreira Ghedini ${ }^{6}$

\section{R E S U M O}

\begin{abstract}
Objetivo: Determinar os fatores prognósticos associados com a recorrência local nos pacientes submetidos a tratamento de sarcoma de tecidos moles em extremidades. Métodos: Foram avaliados retrospectivamente 30 pacientes tratados por ressecção cirúrgica de sarcoma de tecidos moles localizados em extremidades, com seguimento 36,5 $\pm 12,2$ meses. Foram avaliados como fatores prognósticos: idade, sexo, localização, profundidade, localização em compartimentos anatômicos, tamanho, manipulação prévia ao tratamento definitivo, margens cirúrgicas, grau de malignidade histológica, presença de necrose e invasão vascular à histologia. Estes fatores foram correlacionados com a ocorrência de recidiva local do tumor por uma análise univariada e multivariada, sendo considerados significativos valores de $p=0,05$. Resultados: A recorrência local apresentou correlação significativa, tanto na análise univariada como multivariada, com a localização extra-compartimental $(p=0,001)$ e com $o$ alto grau de malignidade $(p=$ $0,024)$. Não houve associação com a idade $(p=1,000)$, com o sexo $(p=1,000)$, com a localização em segmentos corporais $(p=$ $0,544)$, com a profundidade $(p=0,288)$, com o tamanho $(p=0,694)$, com as margens cirúrgicas $(p=0,384)$, com a manipulação prévia $(p=0,461)$, com a presença de necrose $(p=0,461)$ ou invasão vascular $(p=1,000)$. Conclusão: A recorrência local apresentou correlação com a localização extra-compartimental e com o alto grau de malignidade dos tumores.
\end{abstract}

Descritores: Neoplasia. Sarcoma de tecidos moles. Recidiva local de neoplasia.

INTRODUÇÃO

O termo sarcoma de tecidos moles (STM) define um grupo heterogêneo de neoplasias malignas de origem mesenquimal extra-esquelética ${ }^{1,2}$. São relativamente raros e apresentam grande variedade de subtipos histológicos e locais de distribuição pelo corpo, tornando difícil a obtenção de informações consistentes sobre a história natural, prognóstico e tratamento destes tumores 2,3.

O tratamento de escolha dos STM é cirúrgico, complementado ou não pela radio e quimioterapia ${ }^{3}$. A radioterapia adjuvante mostrou-se eficaz na redução de recidivas locais, estimulando a realização de operações conservadoras, reduzindo as amputações para 10 a $20 \%$ dos casos $^{2}$.

Assim como a disseminação metastática, a recidiva local é considerada como falência do tratamento, sendo que vários fatores foram associados com a sua ocorrência como a localização do tumor, o tamanho e o grau de malignidade, mas a qualidade das margens cirúrgicas parece ser o fator mais associado com esta recorrência ${ }^{3-6}$.

O objetivo desse estudo foi correlacionar os fatores prognósticos para recidiva local em pacientes com STM em extremidades.

\section{MÉTODOS}

O estudo foi aprovado pelo Comitê de Ética em Pesquisa da Universidade Federal de Minas Gerais (parecer $n^{\circ}$ ETIC 002/07).

No período de janeiro de 2000 a novembro de 2005 foram atendidos no Serviço de Ortopedia e Traumatologia do Hospital das Clínicas (HC) da Universidade Federal de Minas Gerais (UFMG) e no Biocor Instituto 42 pacientes com diagnóstico de sarcomas extra-esqueléticos localizados em extremidades. Foram excluídos 12, três por não apresentarem dados suficientes no prontuário, três por perderem o seguimento clínico e seis por apresentarem tumores de evolução, tratamento ou prognósticos diferentes, sendo eles o dermatofibrossarcoma $(n=3)$, rabdomiossarcoma $(n=1)$, tumor de Ewing extra-esquelético $(n=1)$ e linfoma nãoHodgkin $(n=1)$.

A idade média dos pacientes foi de $47,66 \pm 19,1$ anos, variando entre 18 e 86 anos. O tempo médio de acompanhamento foi de $36,5 \pm 12,2$ meses, com o mínimo de 19 e o máximo de 62 meses. Dezoito (60\%) eram do sexo masculino e 12 (40 \%) do feminino. O diagnóstico histológico está listado na tabela 1.

Trabalho realizado no Hospital das Clínicas da Universidade Federal de Minas Gerais - UFMG e no Biocor Instituto, Belo Horizonte, MG, Brasil. 1. Coordenador do Ambulatório de Oncologia Ortopédica do Hospital das Clínicas da Universidade Federal de Minas Gerais, Belo Horizonte, MG, Brasil. 2. Professora Associada Doutora do Departamento de Cirurgia da Faculdade de Medicina da Universidade Federal de Minas Gerais, Belo Horizonte, MG, Brasil. 3. Professor Adjunto Doutor do Departamento do Aparelho Locomotor da Faculdade de Medicina da Universidade Federal de Minas Gerais, Belo Horizonte, MG, Brasil. 4. Médico Assistente do Serviço de Ortopedia e Traumatologia do Hospital das Clínicas da Universidade Federal de Minas Gerais Belo Horizonte, MG, Brasil. 5. Mestre em Patologia, Universidade Federal de Minas Gerais, Belo Horizonte, MG, Brasil. 6. Médico residente do Serviço de Ortopedia e Traumatologia do Hospital das Clínicas da Universidade Federal de Minas Gerais, Belo Horizonte, MG, Brasil. 
Tabela 1 - Diagnóstico dos tipos histológicos dos sarcomas de tecidos moles de 30 pacientes submetidos à ressecção cirúrgica no Hospital das Clínicas da UFMG e no Biocor Instituto entre janeiro de 2000 e novembro de 2005.

\begin{tabular}{lccc}
\hline Diagnóstico & Número de pacientes(n) & Frequência Relativa (\%) & Frequência acumulada (\%) \\
\hline Fibrohistiocitoma maligno & 7 & 23,3 & 23,3 \\
Sinoviossarcoma & 7 & 23,3 & 46,6 \\
Lipossarcoma & 4 & 13,3 & 59,9 \\
Fibrossarcoma & 2 & 6,7 & 66,7 \\
Leiomiossarcoma & 2 & 6,7 & 73,4 \\
Neurofibrossarcoma & 2 & 6,7 & 80,1 \\
Sarcoma epitelióide & 2 & 6,7 & 86,8 \\
Angiossarcoma & 1 & 3,3 & 90,1 \\
Sarcoma de células claras & 1 & 3,3 & 93,4 \\
Hemangiopericitoma & 1 & 3,3 & 96,7 \\
Sarcoma de origem indeterminada & 1 & 3,3 & 100 \\
Total & 30 & 100 & 100 \\
\hline
\end{tabular}

Fonte: SAME - HC/UFMG; Biocor Instituto.

Todos os pacientes com suspeita de STM foram submetidos a exame tomográfico ou de ressonância magnética do segmento acometido, tomografia computadorizada de tórax e abdome e cintilografia óssea. O diagnóstico era confirmado por meio de uma biópsia incisional aberta e o material enviado para estudo histopatológico, realizando o perfil imunoistoquímico quando necessário. Os pacientes eram, então, estadiados de acordo com Enneking et al 7 (Tabela 2).

A operação consistia de ressecção em bloco da lesão com margens de tecido normal que envolvia o tumor, do trajeto prévio da biópsia e do orifício de saída do dreno quando presente. A amputação era indicada para tumores volumosos, agressivos, quando não era possível margem adequada, quando o resultado funcional esperado era melhor com a amputação e nos casos infectados e ulcerados. Dos 30 pacientes da amostra 24 (80\%) foram submetidos à operação conservadora com preservação do membro e seis (20\%) foram submetidos à amputações.

Nos pacientes em que a biópsia ou ressecção cirúrgica já havia sido realizada, o diagnóstico era confirmado pela revisão do estudo histopatológico e o estadiamento era complementado. O tratamento consistia de ressecções do tumor residual ou ampliação de margens com a ressecção da cicatriz e de todos os compartimentos envolvidos na operação anterior.

As peças cirúrgicas eram enviadas para estudo histopatológico com definição dos seguintes parâmetros: confirmação do diagnóstico, margens cirúrgicas, grau de malignidade histológica, presença de necrose e invasão vascular. O estudo foi revisto por um único patologista para padronização dos achados histológicos.

O tratamento adjuvante baseava-se em decisão multidisciplinar utilizando como parâmetros o tamanho, a agressividade, a presença de metástases e a qualidade das margens operatórias, e cada tratamento era individualizado, sem um protocolo específico. A radioterapia adjuvante foi realizada em $19(63,3 \%)$ pacientes e a quimioterapia adjuvante em 18 (60\%) pacientes.

O controle pós-operatório consistiu de exame clínico local e sistêmico, tomografia computadorizada de tórax, cintilografia óssea e ressonância magnética do segmento operado. Em caso de recidiva os pacientes eram submetidos à revisão cirúrgica e tratamento complementar quando necessário

Foram avaliados como fatores prognósticos: a idade ( $<50$ anos ou = 50 anos), o sexo, a localização por segmento corporal (membros superiores, membros inferio-

Tabela 2 - Estadiamento de acordo com a classificação proposta por Enneking et al ${ }^{7}$, dos 30 pacientes submetidos ao tratamento cirúrgico no Hospital das Clínicas da UFMG e no Biocor Instituto no período de janeiro de 2000 e novembro de 2005.

\begin{tabular}{lccc}
\hline Enneking & Número de pacientes $(\mathrm{n})$ & Frequência Relativa $(\%)$ & Frequência acumulada (\%) \\
\hline IA & 7 & 23,3 & 23,3 \\
IB & 4 & 13,3 & 36,6 \\
IIA & 5 & 16,6 & 53,3 \\
IIB & 10 & 33,3 & 86,7 \\
III & 4 & 13,3 & 100 \\
Total & 30 & 100 & 100 \\
\hline
\end{tabular}

Fonte: SAME - HC/UFMG; Biocor Instituto. 
res e pelve), a localização em compartimentos (intra ou extra-compartimentais), a profundidade em relação à fáscia muscular (profundos e superficiais), o tamanho do tumor (= $10 \mathrm{~cm}$ ou $>10 \mathrm{~cm}$ ), a manipulação prévia ao tratamento definitivo, as margens cirúrgicas (livres ou comprometidas), o grau de malignidade histológica (alto ou baixo grau), a presença de necrose e a invasão vascular à histologia.

O estudo estatístico foi realizado por uma análise univariada de associação entre a variável resposta (recidiva) e cada umas das variáveis independentes, construindo tabelas de cruzamento tipo 2X2 ou 3X2 e aplicando-se o teste do Qui-quadrado ou o teste exato de Fisher quando uma das frequências era menor que cinco. Foram considerados significativos valores de $p=0,05(5 \%)$. Fatores considerados relevantes $(p<0,250)$ e as variáveis dependentes foram incluídos em uma análise multivariada por regressão logística múltipla. A proporção da variabilidade foi determinada pelo coeficiente de Nagelkerke e o método utilizado na regressão foi o stepwise backward.

Curvas de sobrevida foram construídas para a sobrevida global e recidiva local, utilizando-se o método produto-limite de Kaplan-Meier. A análise foi realizada com o auxílio do software SPSS for Windows ${ }^{\circledR}$ (versão 12.0, (hicago, USA).

\section{RESULTADOS}

No momento do estudo a sobrevida foi de $76,7 \%$ $(n=23)$, sendo que sete $(23,4 \%)$ pacientes evoluíram para óbito, todos em decorrência da disseminação metastática da doença. A recidiva local ocorreu em 10 (33,3\%) pacientes durante o seguimento (Figura 1) com tempo médio de $8,7 \pm 11$ meses. A tabela 3 mostra os resultados da análise univariada para correlação dos fatores prognósticos com a recidiva local.

A recidiva local foi observada em cinco $(29,4 \%)$ pacientes com idade menor de 50 anos e em cinco (38,5\%) com idade igual ou superior a 50, não sendo estatisticamente significativa a diferença entre os grupos $(p=1,000)$. Os pacientes do sexo masculino apresentaram seis $(33,3 \%)$ recidivas locais e do feminino quatro $(33,3 \%)$, sem diferença significativa $(p=1,000)$.

A localização dos STM, de acordo com os segmentos corporais, não mostrou correlação com a recidiva local, sendo que oito (40\%) pacientes com tumores em MMII, um (14\%) paciente com tumor em MMSS e um $(33,3 \%)$ paciente com tumor em pelve apresentaram recidiva local $(p=0,544)$. Todas as recidivas ocorreram em pacientes com tumores profundos, mas a diferença não foi significativa ( $p=0,288)$. Os tumores com localização extra-compartimental apresentaram nove $(64,3 \%)$ recidivas durante o seguimento, enquanto que os tumores de localização intra-compartimental apresentaram um (6,3\%) caso de recidiva local, sendo a diferença significativa $(p=$ $0,001)$.

De acordo com o tamanho do tumor, a média foi de $12,97 \pm 8,6$, sendo que três ( $25 \%$ ) pacientes com tumores menores ou iguais a $10 \mathrm{~cm}$ evoluíram com recidiva local durante o seguimento e sete $(38,9 \%)$ com tumores maiores de $10 \mathrm{~cm}$ apresentaram recidiva do tumor, sem diferença significativa $(p=0,694)$.

Foram observados seis $(27,3 \%)$ casos de recidiva local em pacientes com margens cirúrgicas livres e em quatro (50\%) com margens comprometidas, não havendo diferença significante entre os grupos $(p=0,384)$.

A manipulação cirúrgica prévia não apresentou correlação significativa com a recidiva local, que foi observada em cinco $(27,8 \%)$ pacientes submetidos à manipulação prévia e cinco $(41,7 \%)$ encaminhados antes de qualquer procedimento $(p=0,461)$.

Em relação à análise histológica, o grau de malignidade apresentou correlação estatisticamente significativa com a recidiva local, que ocorreu em nove (50\%) pacientes com tumores de alto grau e em um $(8,3 \%)$ com tumor de baixo grau de malignidade $(p=0,024)$. Nos casos em que havia necrose, cinco (58,3\%) pacientes apresentaram recidiva local, mesmo número observado no grupo sem necrose $(p=0,461)$. A recidiva foi observada em três $(37,5 \%)$ pacientes com invasão vascular e em sete $(31,8 \%)$ sem invasão vascular, não sendo observada diferença significativa $(p=1,000)$.

$\mathrm{Na}$ análise multivariada, o modelo selecionado apresentou coeficiente de Nagelkerke de $51 \%, p=0,70$ no teste de qualidade do ajuste e predição positiva e negativa de $80 \%$. Nesse modelo, o alto grau de malignidade histológica ( $p=0,027 ; R R=4,2)$ e a localização extracompartimental $(p=0,020 ; R R=17,1)$ foram associados ao risco aumentado de ocorrência de recidiva local.

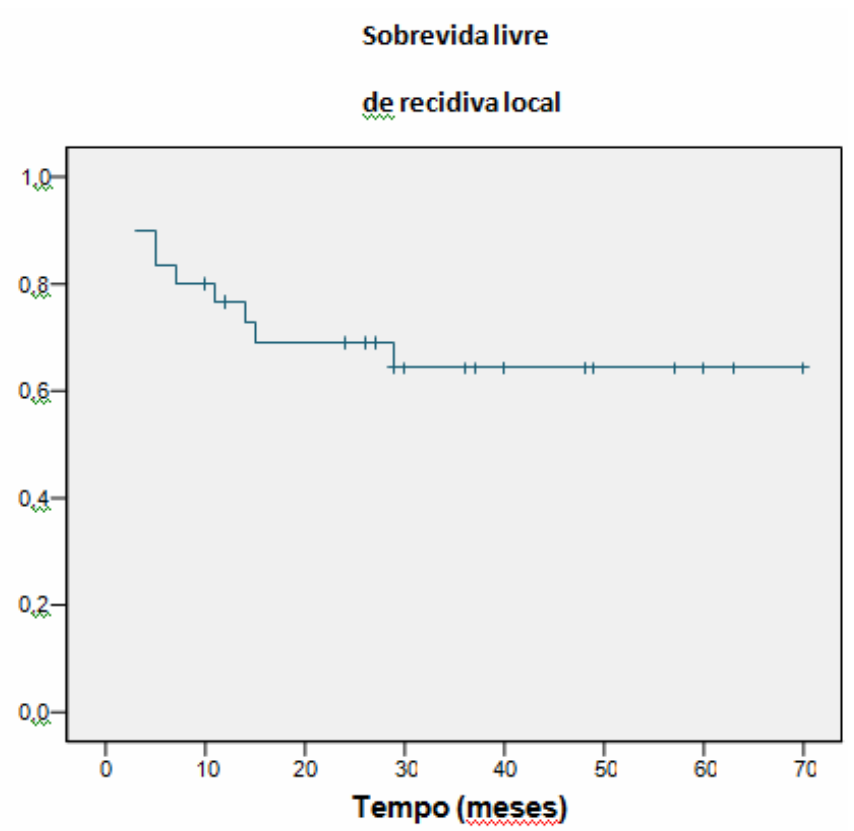

Figura 1 - Curva de sobrevida livre de recidiva local de 30 pacientes submetidos à ressecção cirúrgica no Hospital das Clínicas e no Biocor Instituto no período de janeiro de 2000 a novembro de 2005

Fonte: SAME - HCNFMG; Biocor Instituto 
Tabela 3 - Análise bivariada entre os fatores prognósticos e a recidiva local de 30 pacientes submetidos à ressecção cirúrgica de sarcomas de tecidos moles em extremidades.

\begin{tabular}{|c|c|c|c|c|c|c|}
\hline Fatores Prognósticos & $\mathrm{n}(\%)$ & Recidiva local $n(\%)$ & $p$ & R R & IC $(95 \%)$ & \\
\hline \multicolumn{7}{|l|}{ Idade } \\
\hline$<50$ anos & $17(56,7)$ & $5(29,4)$ & \multirow[t]{2}{*}{1,000} & \multirow[t]{2}{*}{0,60} & 0,40 & - \\
\hline$>50$ anos & $13(43,3)$ & $5(38,5)$ & & & 2,53 & \\
\hline \multicolumn{7}{|l|}{ Sexo } \\
\hline Masculino & $18(60)$ & $6(33,3)$ & \multirow{3}{*}{1,000} & \multirow[t]{3}{*}{2,92} & 0,63 & - \\
\hline Feminino & $12(40)$ & $4(33,3)$ & & & 7,50 & \\
\hline \multicolumn{5}{|l|}{ Localização } & & \\
\hline MMII & $20(66,7)$ & $8(40)$ & \multirow[t]{3}{*}{0,544} & \multirow[t]{3}{*}{1,63} & 0,61 & - \\
\hline MMSS & $7(23,3)$ & $1(14)$ & & & 3,30 & \\
\hline Pelve & $3(10)$ & $1(33,3)$ & & & & \\
\hline \multicolumn{7}{|l|}{ Profundidade } \\
\hline Profundo & $27(90)$ & $10(37)$ & \multirow[t]{3}{*}{0,288} & \multirow[t]{3}{*}{-} & \multirow[t]{2}{*}{ - } & \\
\hline Superficial & $3(10)$ & $0 \quad(0)$ & & & & \\
\hline \multicolumn{5}{|l|}{ Compartimentos } & & \\
\hline Intra-compartimental & $16(53,3)$ & $1 \quad(6,3)$ & \multirow[t]{3}{*}{0,001} & 27,00 & \multirow[t]{3}{*}{2,70} & - \\
\hline Extra-compartimental & $14(46,7)$ & $9(64,3)$ & & 269,46 & & \\
\hline \multicolumn{4}{|l|}{ Tamanho } & & & \\
\hline$=10 \mathrm{~cm}$ & $12(40)$ & $3(25)$ & \multirow[t]{3}{*}{0,694} & \multirow[t]{3}{*}{1,00} & 0,31 & - \\
\hline$>10 \mathrm{~cm}$ & $18(60)$ & $7(38,9)$ & & & 3,28 & \\
\hline \multicolumn{5}{|l|}{ Margem cirúrgica } & & \\
\hline Livre & $22(73,4)$ & $6(27,3)$ & \multirow[t]{3}{*}{0,384} & \multirow[t]{2}{*}{0,71} & 0,19 & - \\
\hline Comprometida & $8(26,6)$ & $4(50)$ & & & 3,14 & \\
\hline \multicolumn{6}{|l|}{ Manipulação prévia } & \\
\hline Sim & $18(60)$ & $5(27,8)$ & \multirow[t]{3}{*}{0,461} & \multirow[t]{2}{*}{1,11} & 0,32 & - \\
\hline Não & $12(40)$ & $5(41,7)$ & & & 3,81 & \\
\hline \multicolumn{6}{|l|}{ Grau histológico } & \\
\hline Alto & $18(40)$ & $9(50)$ & \multirow[t]{2}{*}{0,024} & \multirow[t]{2}{*}{11,00} & 1,64 & - \\
\hline Baixo & $12(60)$ & $1 \quad(8,3)$ & & & 103,94 & \\
\hline Necrose histológica & & & & & & \\
\hline Sim & $12(40)$ & $7(58,3)$ & 0,461 & 1,61 & 0,05 & - \\
\hline Não & $18(60)$ & $5(27,8)$ & & & 65,3 & \\
\hline Invasão vascular histológi & & & & & & \\
\hline Sim & $8(26,6)$ & $3(37,5)$ & 1,000 & 0,70 & 1,22 & - \\
\hline Não & $22(73,4)$ & $7(31,8)$ & & & 170,10 & \\
\hline
\end{tabular}

Legenda: MMSS: membros superiores; MMII: membros inferiores; RR: risco relativo; IC: intervalo de confiança Fonte: SAME - HC/UFMG; Biocor Instituto

\section{DISCUSSÃO}

O tratamento local dos STM realizado com operação conservadora e radioterapia, tem alcançado taxas de recidiva local de 10 a $20 \%$ 23,5. Apesar da radioterapia como adjuvante no controle local da doença, a sua indicação não é uniforme na literatura ${ }^{3,5,6,8-11}$.

Nesta série, a recidiva local foi observada em $1 / 3$ dos pacientes, ocorrência discretamente mais alta que a maioria das séries estudadas, que apresentam $14,5 \%$ a $27 \%$ de recorrência $2,3,6,8,9$. A provável causa para maior incidência de recorrência local foi o maior tamanho dos tumores encontrados nesta casuística com muitos tumores de localização extra-compartimental $(46,7 \%)$, o que dificulta a aquisição de controle cirúrgico local adequado.
A localização extra-compartimental foi importante fator prognóstico para a ocorrência de recidiva local. Os tumores considerados extra-compartimentais usualmente apresentam tratamento cirúrgico mais difícil e margens mais exíguas, já que estão em íntima relação com estruturas neurovasculares. Tanto a dificuldade de alcançar margens adequadas, quanto a localização em regiões muito vascularizadas e ricas em drenagem linfática provavelmente facilitam a disseminação desses tumores e a recorrência local, podendo explicar os resultados obtidos.

$\mathrm{O}$ alto grau de malignidade mostrou correlação com a recidiva local, sendo que dos casos recorrentes $90 \%$ eram de alto grau. A maioria dos estudos mostra que o alto grau de malignidade tem forte influência no desenvolvimento de metástases e na recidiva local, sendo um dos 
mais importantes fatores independentes relacionados com a agressividade dos STM 2-6, 9,10.

Apesar de $50 \%$ dos pacientes que apresentavam margens comprometidas desenvolverem recidiva, não foi observada correlação com a recidiva local. A escolha do tipo de margem (livre ou contaminada) parece ser o fator responsável por esse achado. De acordo com Kawaguchi et al. ${ }^{11}$, a simples seleção de margens livres no exame histopatológico não é capaz de predizer a recorrência local, sendo a definição de margens curativas ainda controversa. O tempo de seguimento também pode ter influência neste achado. Engelau et al. ${ }^{12}$ e Stojadinovic et al. ${ }^{6}$, observaram que os fatores prognósticos apresentam influência variável de acordo com o tempo de seguimento, sendo que as margens cirúrgicas apresentam valor prognóstico mais tardiamente no seguimento.

Outro fator que não influenciou na recidiva foi a manipulação prévia do tumor, sugerindo, em concordância com outros autores ${ }^{13,14}$, que o tratamento definitivo com ampliação adequada das margens de um tumor manipulado previamente pode evitar disseminação local do sarcoma, desde que seja realizado em tempo hábil.

Em conclusão, o alto grau de malignidade à histologia e a localização extra-compartimental apresentaram correlação com a recidiva local nos STM em extremidades.

\section{A B S T R A C T}

Objective: To determinate the prognostic factors associated with the local relapse in patients treated for soft tissue sarcoma in extremities. Methods: Retrospectively, 30 patients were evaluated after being treated with surgical resection of soft tissue sarcoma located in extremities, with a outcome of 36,5 $\pm 12,2$ months. The prognostic factors evaluated were: age, sex, location, depth, location in the anatomic compartments, size, tumor previous manipulation, surgical margins, grade of histological malignance, presence of histological necrosis or histological vascular invasion. These factors were correlated with the local relapse of the tumor with a univariated and multivariated analysis with significance when $p=0,05$. Results: Local relapse presented significant correlaction in univariated and multivariated analysis with extra-compartimental location $(p=0,001)$ and with high grade histological malignance $(p=0,001)$. There was no significant association with age $(p=1,000)$, sex $(p=1,000)$, body location $(p=0,544)$, depth $(p=0,288)$, size $(p=0,694)$, surgical margins $(p=0,384)$, previous manipulation $(p=0,461)$, necrosis $(p=0,461)$ or with the presence of vascular invasion $(p=1,000)$. Conclusion: Local relapse presents correlaction with extra-compartimental location and with high grade malignancy tumors.

Key words: Neoplasms. Soft tissue sarcoma. Neoplasm recurrence.

\section{REFERÊNCIAS}

1. Enzinger F.M., Weiss S.W.: Soft Tissue Tumors. Missouri: MosbyYear Book; 1995.

2. Ishihara HY, Jesus Garcia R, Korukian M, Ponte FM. Sarcoma de tecidos moles: fatores prognósticos. Rev Bras Ortop. 2004; 39(11/ 12): $637-47$

3. Engellau J. Prognostic factors in soft tissue sarcoma. Acta Orthop Scand. (Suppl 314) 2004; 75: 1 - 52.

4. Rydholm, Prognostic factors in soft tissue sarcoma. Acta Orthop Scand. (supl.273) 1997; 68: 148 -55.

5. Trovik CS. Local recurrence of soft tissue sarcoma. A Scandinavian Sarcoma Group Project. Acta Orthop Scand. (Suppl 300) 2001; 72: $1-31$.

6. Stojadinovic A, Leung DHY, Lewis JJ, Jaques DP, Brennan MF. Primary adult soft tissue sarcoma: time-dependent influence of prognostic variables. J Clin Oncol. 2002; 20(21): 4344 - 52.

7. Enneking WF, Spanier SS, Goodman MA. A system for the surgical staging of musculoskeletal sarcoma. Clin Orthop. 1980; 153: 106 -20 .

8. Leidinger $B$, Heyse $T$, Schuck $A$, Buerger $H$, Mommsen $P$, Bruening $T$, et al. High incidence of metastatic disease in primary high grade and large extremity soft tissue sarcomas treated without chemotherapy. BMC Cancer 2006; 6: 160, (www.biomedcentral.com/1471-2407/6/160).

9. Stojadinovic A, Leung DHY, Hoos A, Jaques DP, Lewis JJ, Brennan MF. Analysis of the prognostic significance of microscopic margins in 2,084 localized primary adult soft tissue sarcomas. Ann Surg. 2002; 235(3): $424-34$

10. Coindre JM, Terrier P, Bui NB, Bonichon F, Collin F, Le Doussal V, et al. Prognostic factors in adult patients with locally controlled soft tissue sarcoma. A study of 546 patients from the French Federation of Cancer Centers Sarcoma Group. J Clin Oncol. 1996; 14: 869 77 .

11. Kawaguchi N, Ahmed AR, Matsumoto S, Manabe J, Matsushita Y. The concept of curative margin in surgery for bone and soft tissue sarcoma. Clin Orthop. 2004; 419: 165 - 72.

12. Engelau J, Anderson H, Rydholm A, Baures HC, Hall KS, Gustafson $P$, et al. Time dependence of prognostic factors for patients with soft tissue sarcoma: a Scandinavian Sarcoma Group Study of 338 malignant fibrous histiocytomas. Cancer 2004; 100 (10): 2233 -39 .

13. Zornig C, Peiper M, Scroder S. Re-excision of soft tissue sarcoma after inadequate initial operation. Br J Surg. 1995; 82 (2): 278 79

14. Manoso MW, Frassica DA, Deune EG, Frassica FJ. Outcomes of reexcision after unplanned excisions of soft-tissue sarcomas. J Surg Oncol. 2005; ;91 (3):153-58.

Recebido em 05/12/2008

Aceito para publicação em 02/02/2009

Conflito de interesse: nenhum

Fonte de financiamento: nenhuma

\section{Como citar esse artigo:}

Teixeira LEM, Araújo ID, Andrade MAP, Gomes RA, Salles PGO, Ghedini DF. Recidiva local nos sarcomas de tecidos moles: fatores prognósticos. Rev Col Bras Cir. [periódico na Internet] 2009; 36(5). Disponível em URL: http://www.scielo.br/rcbc

Endereço para correspondência:

Luiz Eduardo M Teixeira

E-mail: luizmteixeira@yahoo.com.br 